\title{
Electron Capture Dissociation of Triantennary Complex-Type $N$-Glycosylated Peptides: A Case of Suppressed Peptide Backbone Cleavage
}

\author{
Yayoi Hongo, ${ }^{1 *}$ Takemichi NakAmuRa, ${ }^{2}$ and Akihiro SAto ${ }^{3}$ \\ ${ }^{1}$ Molecular Characterization Team, RIKEN, Wako, SAITAMA, JAPAN \\ ${ }^{2}$ Biomolecular Characterization Team, RIKEN, Wako, SAITAMA, JAPAN \\ ${ }^{3}$ JASCO International Co., Ltd., Hachioji, TOKYO, JAPAN
}

\begin{abstract}
We investigated the electron capture dissociation (ECD) of triantennary complex-type $N$-glycosylated peptides prepared from bovine fetuin. Previous reports on Fourier-transform ion cyclotron resonance mass spectrometry (FT-ICR-MS) suggested that ECD is an advantageous method for structural characterization of glycopeptides, because it can cleave the $\mathrm{N}-\mathrm{C} \alpha$ bond on the peptide backbone while retaining the labile glycosidic bonds. We present here new data from ECD which resulted in the degradation of the glycan structure prior to any backbone cleavage. Based on the ECD experiment on deglycosylated samples, the character of the peptide backbone sequence, which contains carbamoylmethylated cysteine residue, seemed to prevent extensive $\mathrm{N}-\mathrm{C} \alpha$ bond cleavage. It appears that more basic investigations are necessary to promote practical use of ECD for structural characterization of complex glycopeptides because each type of glycopeptide exhibits particular fragmentation patterns.
\end{abstract}

(Received November 13, 2006; Accepted December 7, 2006)

\section{Introduction}

Tandem mass spectrometry is a powerful technique for structural characterization of proteins and peptides. In particular, it is advantageous to use Fourier-transform ion cyclotron resonance mass spectrometry (FTICR-MS), which can trap ions in an analyzer cell, and then examine different activation methods including "sustained off-resonance irradiation" collision induced dissociation (SORI-CID), infrared multiphoton dissociation (IRMPD), and electron capture dissociation (ECD) (reviewed in Selno and Volmer ${ }^{1)}$ ). SORI-CID and IRMPD are well known as "slow-heating" techniques which result in selective bond cleavage at the most labile site in the gas phase. On the other hand, ECD is a relatively new method, ${ }^{2,3)}$ where low energy electrons react with multiply charged peptides in the gas phase. Then, the $\mathrm{N}-\mathrm{C} \alpha$ bond on the glycopeptide backbone can be cleaved to give extensive "c- and z-type" ions while retaining the labile glycosidic bonds intact. ECD is expected to have the capability to provide structural information on glycopeptides complementary to the information from IRMPD, so that combination of ECD with IRMPD may become an effective tool for glycopeptide characterization. Håkansson et $a l_{.}{ }^{4), 5)}$ and Adamson and Håkansson ${ }^{6)}$ have studied ECD on $N$ linked glycopeptides and observed extensive backbone cleavage (except for the $N$-terminal side of proline) without cleavage of glycosidic bonds. Kjedsen et al. ${ }^{7)}$ also reported extensive $\mathrm{N}-\mathrm{C} \alpha$ cleavage of protein con-

* Correspondence to: Yayoi Hongo, Molecular Characterization Team, RIKEN (Inst. Phys. Chem. Res.), 2-1 Hirosawa, Wako, Saitama 351-0198, JAPAN, e-mail: yayoi@riken.jp taining $N$ - and $O$-linked glycans. However, in the latter case, preferential $N$-acetylneuraminic acid (NeuNAc) losses from the glycan structure were also observed. Recently, Mormann et al. ${ }^{8)}$ explored ECD on $O$ glycosylated peptide and also demonstrated loss of acetyl radicals from the $N$-acetyl group of the $N$ acetylhexose (HexNAc) with fragments corresponding to peptide backbone cleavage. These previous studies suggested that different types of glycopeptide exhibit different fragmentation patterns. To facilitate the use of ECD in the structural characterization of complex glycopeptides, more basic investigations are necessary. We present here a new data set of ECD spectra of complex-type triantennary $N$-glycosylated peptide. To the best of our knowledge, the glycan structure in our glycopeptide samples from bovine fetuin (Fig. 1) is the most complex and largest structure; a triantennary glycan of nearly $3 \mathrm{kDa}(2,861 \mathrm{u})$, for which ECD has never been reported. It turned out that ECD experiments on complex-type triantennary $N$-glycosylated peptides did not result in backbone $\mathrm{N}-\mathrm{C} \alpha$ bond cleavage but exhibited preferential cleavage of terminal glycoside units, $N$-acetylneuraminic acid (NeuNAc), and losses of some radicals ( $-43 \mathrm{u},-58 \mathrm{u}$, and $-90 \mathrm{u}$ ). In this paper, we describe ECD of glycopeptides that did not result in backbone fragmentation. Our data indicate that the ECD process is regulated by multiple factors.

\section{Experimental}

The triantennary complex-type $N$-glycosylated tryptic peptides were prepared from commercially available fetuin (Wako Chemicals). Ammonium bicarbonate, formic acid (amino acid sequencing-grade), iodoacet- 


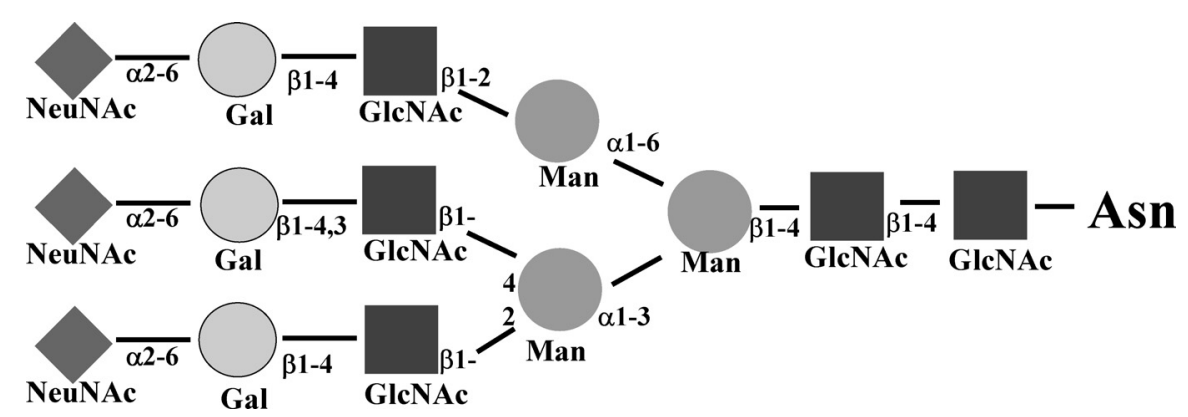

Fig. 1. Schematic representation of triantennary complex-type $N$-linked glycopeptide.

$\diamond, N$-Acetylneuraminic acid (NeuNAc); $\bigcirc$, Galactose (Gal); $\bigcirc$, Mannose (Man); $\boldsymbol{\square}, N$-Acetyl-D-glucosamine (GlcNAc).

Table 1. Amino Acid Sequences and Monoisotopic Mass of Two Triantennary Complex-Type $N$-Glycopeptides and Two Deglycosylated Peptides

Glycan structure linked to $\mathrm{N}^{*}$. Exact mass of glycan is 2861.000. Enzymatic deglycosylation substitute Asn with Asp. Cysteine is carbamoylmethylated $\left(\mathrm{C}^{* *}\right)$.

\begin{tabular}{clc}
\hline Sample & \multicolumn{1}{c}{ Sequence } & Monoisotopic mass \\
\hline GP1 & L C **P D C** P L L A P L N* D S R & 4600.833 \\
GP2 & R P T G E V Y D I E I D T L E T T C** H V L D P T P L A N* C** S V R & 6531.761 \\
Deglycosylated & L C** P D C** P L L A P L D D S R & 1739.833 \\
GP1 & R P T GE V Y D IE I D T LE T T C** H V L D P T P L A D C** S V R & 3670.761 \\
Deglycosylated & GP2 &
\end{tabular}

amide, dithiothreitol (DTT), and distilled purified water were also purchased from Wako. Trypsin, PNGase $\mathrm{F}$, and neuraminidase were purchased from Sigma. RapiGest SF (lyophilized sodium-3-[(2-methyl-2-undecyl-1,3-dioxolan-4-yl)-methoxyl]-1-propanesulfonate) was obtained from Waters. Prior to tryptic digestion, $\mathrm{S}-\mathrm{S}$ bonds in fetuin were reduced by DTT and alkylated with iodoacetamide (powder) in 0.1\% RapiGest SF solution. ${ }^{9)}$ Tryptic digestion of carbamoylmethylated fetuin was performed at an enzyme-to-protein molar ratio of $1: 100$. Removal of the glycan part was carried out by enzymatic digestion with PNGase F from Chryseobacterium (Flavobacterium) meningsepticum (SigmaAldrich). The enzyme-to-protein molar ratio was 1 : $1,400,000$ (25 units of the enzyme were used). ${ }^{10)}$ Glycosylated asparagine residues in the peptides were converted to aspartic acid residues by PNGase F digestions.

Off-line separation and concentration of glycopeptide was carried using a $\mathrm{C}_{4}(4.6 \mathrm{~mm} \phi \mathrm{UG}-5)$ column (Nomura Chemical Co.) with a flow rate of $1.0 \mathrm{~mL} / \mathrm{min}$ at room temperature. The elution conditions for separation were solvent $\mathrm{A}(0.1 \%$ formic acid in water, $\mathrm{w} / \mathrm{w})$ plus $2 \%$ solvent $\mathrm{B}\left(\mathrm{CH}_{3} \mathrm{CN}\right), \mathrm{v} / \mathrm{v}$, for the first $2 \mathrm{~min}$, followed by a linear gradient to $60 \% \mathrm{~B}$ in $58 \mathrm{~min}$, then to $80 \% \mathrm{~B}$ in $72 \mathrm{~min}$. Electrospray ionization mass spectrometry was performed with a T100LC (JEOL). The instruments were operated in positive ion mode, tuned for the $m / z$ range of 200-2500. Operating conditions optimized for the detection of peptides were as follows: capillary voltage $1,800 \mathrm{~V}$ and sample cone voltage $7 \mathrm{~V}$. Amino acid sequences and monoisotopic mass of samples are listed in Table 1 . Observed $\mathrm{m} / \mathrm{z}$ values for peptide samples are consistent with previous reports for triantennary complex-type glycostructure $^{11), 12)}$ (shown in Fig. 1).
A QFT-7 (Quadrupole Fourier Transform, IonSpec FTMS systems, Varian Inc.) mass spectrometer equipped with a nano-ESI source was used for the ECD experiments. The fetuin peptide samples were electrosprayed from a solution in $1: 1$ acetonitrile/water with $0.1 \%$ formic acid at a flow rate of $0.1 \mu \mathrm{L} / \mathrm{min}$. The most abundant charge state for each sample, e.g., triplecharge protonated molecules for GP1 and quadruplecharge protonated molecules for GP2, was selected as the precursor ion for the ECD experiments. In this study, the precursor ions were isolated with the quadrupole mass filter (called Q2 in this particular instrument) to avoid overfilling of the ion cyclotron resonance (ICR) cell with unwanted ions. In the typical experimental setting, the precursor ions were separated by Q2 (set with a separation window of a few $\mathrm{m} / \mathrm{z}$ to allow all of the isotope peaks through) and accumulated in the Q3 hexapole for $5 \mathrm{~s}$ to maximize the signal. Then, the accumulated ions were pulsed into the ICR cell, followed by a $150 \mathrm{~ms}$ delay and a gas pulse to cool down the ions. After another $150 \mathrm{~ms}$ delay, electrons emitted from a directly heated dispenser cathode $^{13), 14)}$ at the back of the ICR cell were introduced into the cell for $100 \mathrm{~ms}$ at a $1.5 \mathrm{eV}$ energy. The product ions were excited after a delay (750-4,750 ms) to acquire broadband spectra with Omega 8 software. The ECD conditions were essentially the same as those optimized for substance P. A longer electron irradiation, e.g., $300 \mathrm{~ms}$, did not alter the cleavage site by ECD. The electron energies stated here are not precisely calibrated values but nominal ones based on the electrode potentials as in most ECD experiments using commercial instruments. 


\section{Results and Discussion}

Backbone cleavage was not observed in ECD spectra

Figure 2 shows the results of ECD on triple-charged $N$-glycosylated peptide GP1 (LCPDCPLLAPLN-glyDSR $\left.[\mathrm{GP} 1+3 \mathrm{H}]^{3+}\right)$. ECD resulted in glycan degradation (GlcNAc-GlcNAc, NeuNAc-Gal, and GlcNAc-Man cleavage), whereas no backbone cleavage to generate "c-, z-type" ions was observed. ECD also yielded the charge-reduced radical ion $[\mathrm{GP} 1+3 \mathrm{H}]^{2+}$ and loss of NeuNAc, $43 \mathrm{u}, 58 \mathrm{u}$, and $90 \mathrm{u}$ (the latter two neutrals presumably contain one nitrogen, respectively) from $[\mathrm{GP} 1+3 \mathrm{H}]^{2+}$. The double-charged product ions around $m / z 2156.8$ are attributable to the loss of $292 \mathrm{u}$ (a radical corresponding to NeuNAc) from the chargereduced precursor ion $[\mathrm{GP} 1+3 \mathrm{H}]^{2+}$. The triplecharged product ions around 1437.6 can be generated by the loss of $291 \mathrm{u}$ (a neutral corresponding to NeuNAc) from the triple-charged precursor ion [GP1+ $3 \mathrm{H}]^{3+}$. Kjedsen et al. $^{7)}$ have previously reported that some NeuNAc, which is a terminal end glycan, are removed by ECD. Mormann et al. ${ }^{8)}$ have also observed glycan degradation combined with extensive "c-, z-type" ions. However, in our present case, "c-, z-type" ions were not observed but glycan degradation and radical losses were dominant. Figure 3 shows ECD spectra of quadruple-charge protonated GP2 (RPTGEVYDIEIDTLETTCHVLDPTPLAN-glyCSVR [GP2+ $\left.4 \mathrm{H}]^{4+}\right)$. ECD resulted in charge reduction products $[\mathrm{GP} 2+4 \mathrm{H}]^{3+\cdot}$ and $[\mathrm{GP} 2+4 \mathrm{H}]^{2+\cdot}$. Presumed losses of NeuNAc $(291 \mathrm{u})$ from the charge reduction products were dominant; however, no "c-, $z$-type" fragment ions attributable to backbone cleavage were observed. In addition, as in the case of GP1, ECD on GP2 also yielded small radical losses $(-43 \mathrm{u},-58 \mathrm{u}$, and $-90 \mathrm{u})$ from $[\mathrm{GP} 2+4 \mathrm{H}]^{3+}$. The presence of charge reduction products showed that electron capture $(1.5 \mathrm{eV})$ successfully occurred. Mormann et al. ${ }^{8)}$ reported predominant loss of $\cdot \mathrm{C}_{2} \mathrm{H}_{3} \mathrm{O}(43 \mathrm{u})$ from HexNAc in the ECD spectra of $O$-glycopeptide due to the attack of hydrogen at the nitrogen of the $N$-acetyl group. Glycopeptides GP1 and GP2 also contained GlcNAc groups in their structures; therefore, $-43 \mathrm{u}$ can be attributed to the loss of acetyl radical. On the other hand, $-58 \mathrm{u}$ and $-90 \mathrm{u}$ observed in the present ECD spectra were characteristic of both GP1 and GP2. The measured mass differences were consistent with the loss of $\cdot \mathrm{CH}_{2} \mathrm{CONH}_{2}(58 \mathrm{u})$ and - $\mathrm{SCH}_{2} \mathrm{CONH}_{2}(90 \mathrm{u})$, respectively. GP1 and GP2 did not contain either Asn or Gln; therefore, the loss of - $\mathrm{CH}_{2} \mathrm{CONH}_{2}(58 \mathrm{u})$ could not come from side-chain fragmentation of amino acid.

In the next section, we try to clarify the factor that thwarted extensive peptide backbone cleavage, i.e., whether it is the character of the glycan or that of the peptide.

Contribution of peptide backbone character to ECD fragmentation

A successful electron capture event was confirmed by the observation of charge reduction products. Therefore, characteristic radical losses $(-58 \mathrm{u}$ and $-90 \mathrm{u}$ ) and no backbone fragmentation were expected to be caused by chemical structures of GP1 and GP2. Glycopeptides GP1 and GP2 have the same glycan structure but different amino acid sequences. Both glycopeptides contain Leu, Pro, Asp, Ala, Ser, Arg, and carbamoylmethyl modified Cys in common. In order to

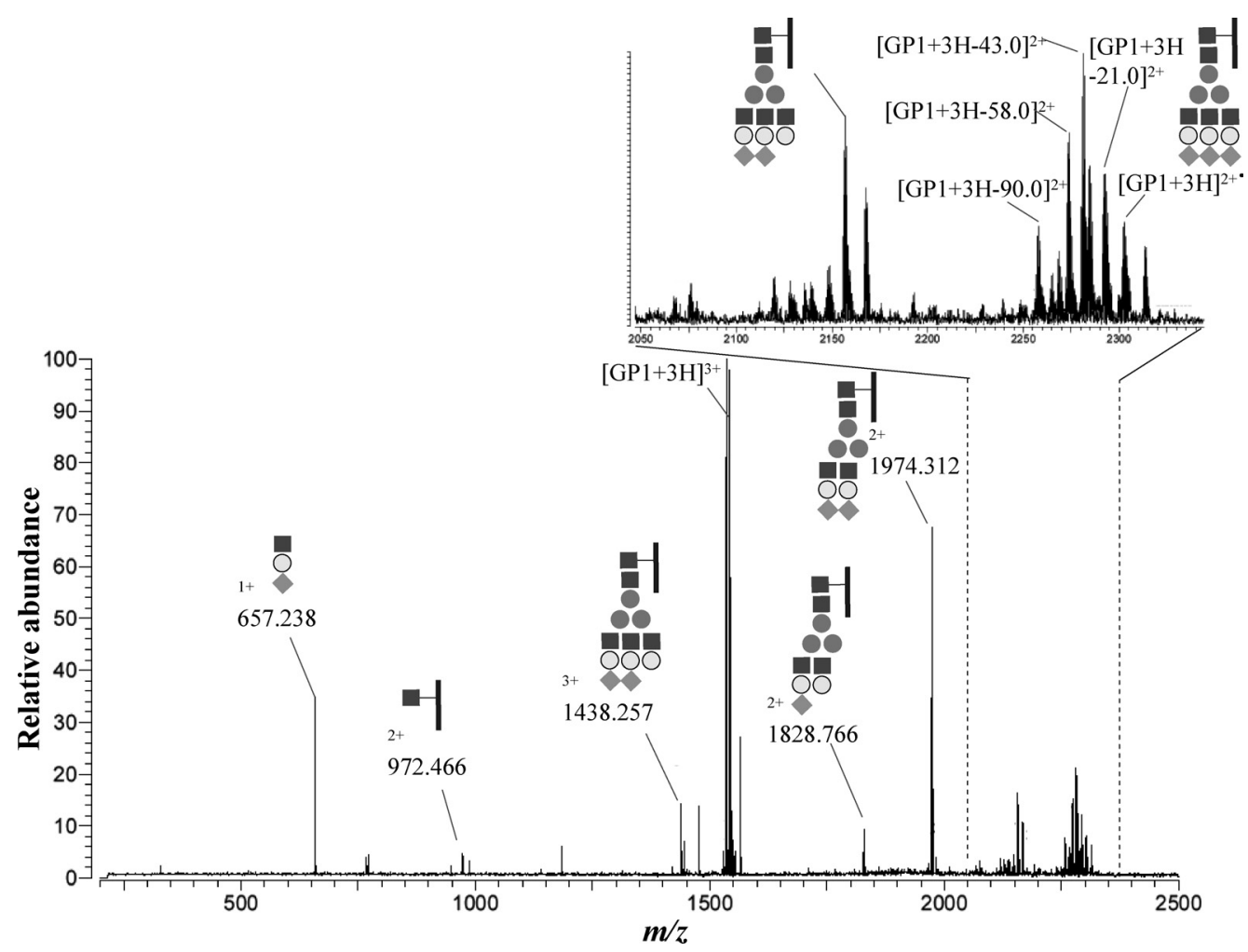

Fig. 2. ECD spectrum of glycopeptide GP1. $[\mathrm{GP} 1+3 \mathrm{H}]^{3+}$ was selected as a precursor ion. The spectrum showed degradation of the glycan unit and small radical losses $(21 \mathrm{u}, 43 \mathrm{u}, 58 \mathrm{u}$, and $90 \mathrm{u})$ from charge reduced product $\left([\mathrm{GP} 1+3 \mathrm{H}]^{2+\cdot}\right)$. 


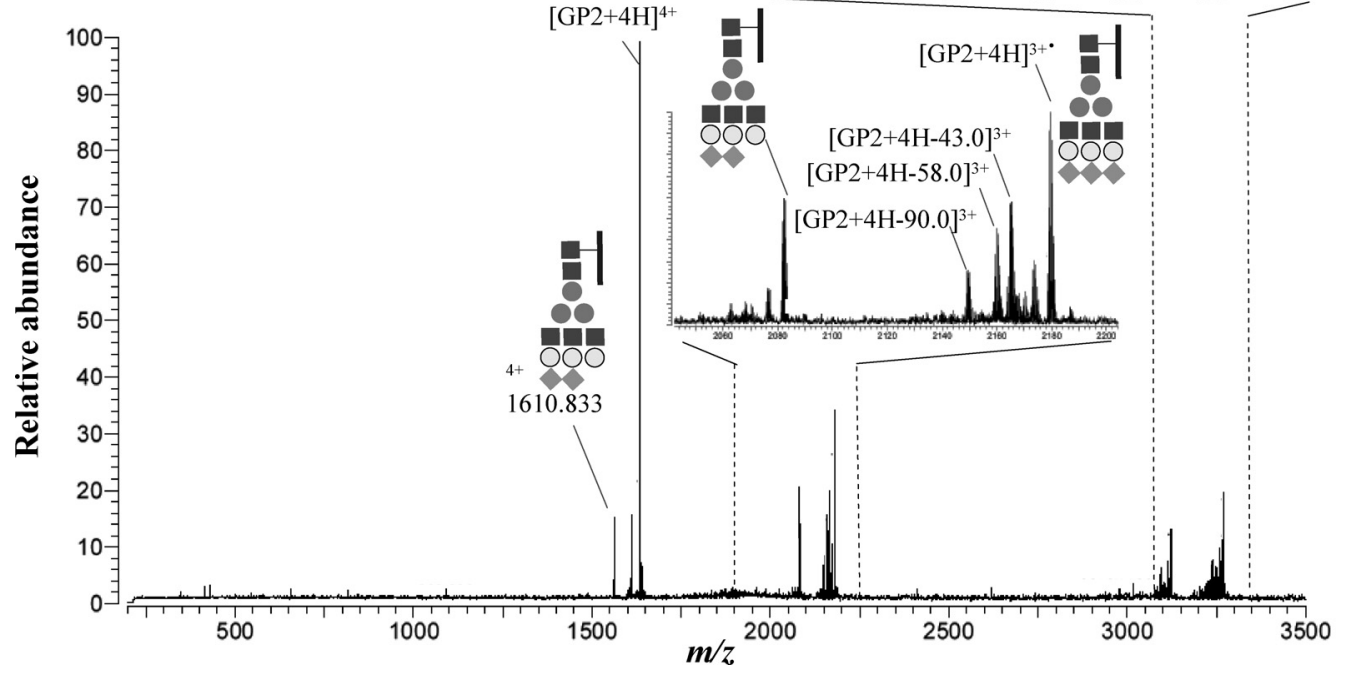

Fig. 3. ECD spectrum of glycopeptide GP2. $[\mathrm{GP} 2+4 \mathrm{H}]^{4+}$ was selected as a precursor ion. The spectrum showed two charge reduced products, $[\mathrm{GP} 2+4 \mathrm{H}]^{3+\cdot}$ and $[\mathrm{GP} 2+4 \mathrm{H}]^{2+\cdot \cdot}$, and small radical $(43 \mathrm{u}, 58 \mathrm{u}$, and $90 \mathrm{u})$ and NeuNAc losses.

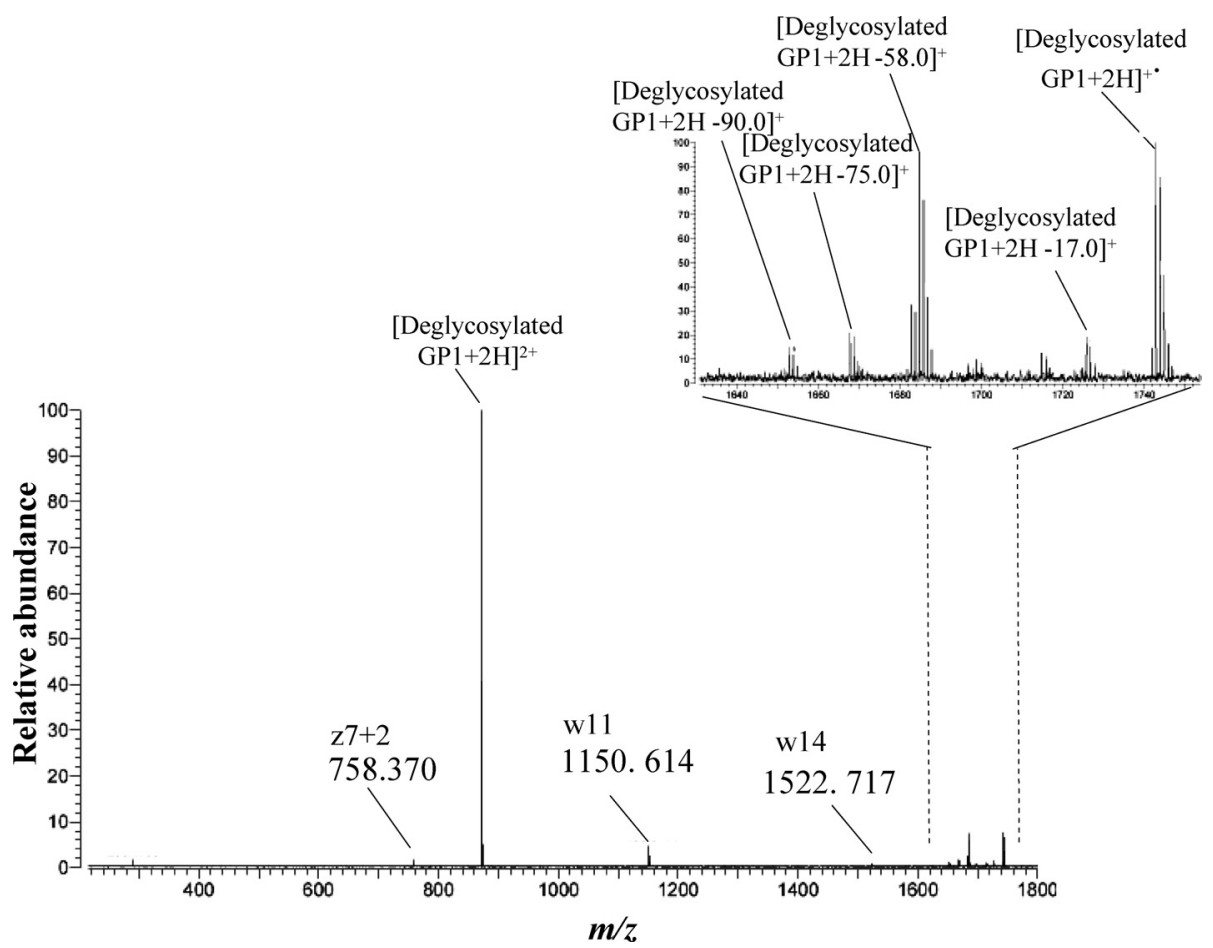

Fig. 4. ECD spectrum of doubly charged precursor ion [deglycosylated-GP1 $+2 \mathrm{H}]^{2+}$. Three product ions arose from backbone cleavage, namely $\mathrm{z}_{7}+2, \mathrm{w}_{11}$, and $\mathrm{w}_{14}$.

observe the ECD fragmentation of the deglycosylated peptide backbone, enzymatic removal of sugar chains was performed. PNGase F digestion of the glycopeptides gave sugar-free peptides; each has an Asp residue at the site where the glycosylated Asn originally was present. Figure 4 shows the ECD spectrum of [deglyco-
sylated-GP $1+2 \mathrm{H}^{2+}$. Barely three $C$-terminal side fragment ions, $z_{7}+2, \mathrm{w}_{11}$, and $\mathrm{w}_{14}$, arose from backbone cleavage. The two "w-type" ions were specific to the cleavage at the $N$-terminal side of each cysteine residue. In addition, $58 \mathrm{u}$ and $90 \mathrm{u}$ losses from [deglycosylated-GP1 $1+3 \mathrm{H}]^{2+\cdot}$ were observed with the charge- 


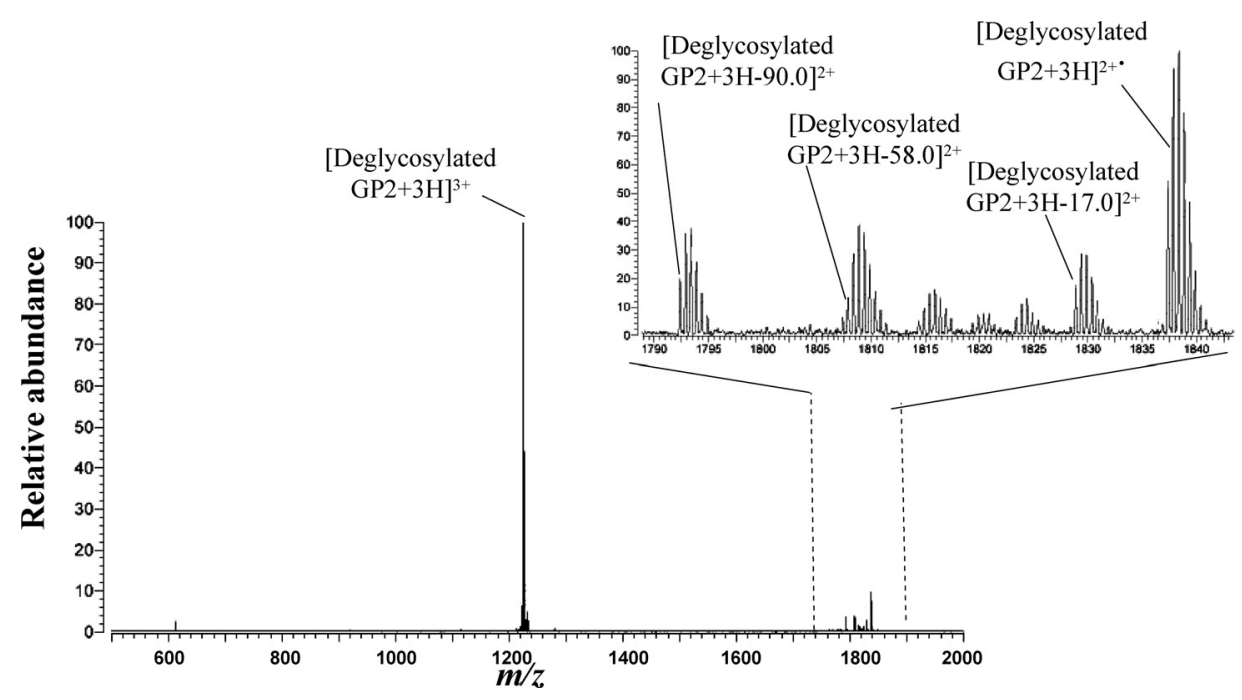

Fig. 5. ECD spectrum of triply charged precursor ion [deglycosylated-GP $2+3 \mathrm{H}]^{3+}$. No ions arose from backbone cleavage.

reduced species, in a similar manner to the previous experiments for glycopeptides. This observation suggested that the lack of backbone cleavage in the glycopeptide was due to the nature of the peptide sequence and/or amino acid composition. Figure 5 shows the ECD for [deglycosylated-GP $2+3 \mathrm{H}]^{3+}$, which also did not give extensive backbone cleavage. In all of the ECD experiments, presumed radical losses $(-58 \mathrm{u}$ and $-90 \mathrm{u})$ from the charge reduction products were observed and these losses were assigned to the side-chain cleavages from carbamoylmethylated cysteine residues, on the basis of measured masses. Structural information from ECD was still limited even after deglycosylation.

We have shown that ECD did not give peptide backbone cleavage in the present complex-type glycopeptides GP1 and GP2. ECD has been regarded as the technique that cleaves the $\mathrm{N}-\mathrm{C} \alpha$ bond in peptide backbones rather more non-selectively and/or uniformly than "slow-heating" techniques. ${ }^{1-8)}$ However, in the past two or three years, some evidence has been reported that ECD does not always give uniform $\mathrm{N}-\mathrm{C} \alpha$ bond cleavage. For example, Tsybin et al. ${ }^{15)}$ showed that the helical structure of the peptide ion induces periodic abundance of "c-, $z$-type" fragments from peptide $\mathrm{N}-\mathrm{C} \alpha$ bond cleavage in ECD. Our finding is another example that ECD does not always result in non-selective and extensive $\mathrm{N}-\mathrm{C} \alpha$ bond cleavage. ECD is not controlled in a simple way as stated earlier. ${ }^{2)-5)}$ Leymarie et al. ${ }^{16)}$ pointed out that a "mobile radical" is indispensable to produce a relatively long-lived intermediate radical, which induces extensive backbone cleavage in ECD. The importance of the "mobile radical" for extensive cleavage was also proposed by Belyayev et al. ${ }^{17)}$ They confirmed that coumarin modified substance $\mathrm{P}$ also reduces backbone fragmentation but promotes sidechain cleavage upon ECD due to the radical localization on coumarin. In this work, carbamoylmethylated cysteine residue seemed to serve as a "radical trap"17) and induced losses of $58 \mathrm{u}$ and $90 \mathrm{u}$. Although some other experiments and theoretical calculations have already shown that ECD readily cleaves disulfide bonds, the reason why the hydrogen radical localizes to sulfur is still a matter of debate. ${ }^{18)-21)}$ Sulfur in cysteine residues may hold hydrogen and prevent its migration to the peptide backbone. If this hypothesis is correct, then we should be able to enhance backbone cleavage in glycopeptides by removing the Cys side-chain or by modifying Cys to a derivative that does not allow hydrogen radical localization.

In previously reported studies ${ }^{4)-8)}$ on ECD of glycopeptides, high-mannose-type, xylose-type, and complex-type glycopeptides have shown extensive $\mathrm{N}-\mathrm{C} \alpha$ bond cleavage on the peptide backbone without glycan degradation. Coincidently, glycopeptide samples in those previous studies have no cysteine residues in their backbone sequences and no NeuNAc in the main glycan structure. This is in sharp contrast to our triantennary complex-type glycopeptides, which include both Cys and NeuNAc. The data presented here suggest that sequencing of glycopeptides with ECD may not be so straightforward and depends on their structure.

In summary, we have shown that some glycopeptides are resistant to backbone cleavage upon ECD. It appears that the nature of the backbone peptide itself prevents extensive $\mathrm{N}-\mathrm{C} \alpha$ bond cleavage. Current observations support the view that ECD is regulated by multiple factors. The solution to this problem is currently under investigation.

\section{Acknowledgements}

This research was partially supported by the Ministry of Education, Science, Sports and Culture, Grant-inAid for Scientific Research (C), 18550085, 2006, and RIKEN Special Project Funding for Basic Science (Chemical Biology Research Project). The authors thank JASCO International Co. for their support.

\section{References}

1) L. Selno and D. A. Volmer, J. Mass Spectrom., 39, 1091 (2004).

2) R. A. Zubarev, N. L. Kelleher, and F. W. McLafferty, J. Am. Chem. Soc., 120, 3265 (1998). 
3) R. A. Zubarev, Current Opinion Biotech., 15, 12 (2004).

4) K. Håkansson, H. J. Cooper, M. R. Emmett, C. E. Costello, A. G. Marshall, and C. L. Nilsson, Anal. Chem., 73, 4530 (2001).

5) P. Håkansson, M. J. Chalmers, J. P. Quinn, M. A. McFarland, C. L. Hendrickson, and A. G. Marshall, Anal. Chem., 75, 3256 (2003).

6) J. Adamson and K. Håkansson, J. Proteome, 5, 493 (2006).

7) F. Kjedsen, K. F. Haselmann, B. A. Budnik, E. S. Sørensen, and R. A. Zubarev, Anal. Chem., 75, 2355 (2003).

8) M. Mormann and H. Paulsen, Eur. J. Mass Spectrom., 11, 497 (2005)

9) T. Imre, G. Schlosser, G. Pocsfalvi, R. Siciliano, E. Molnar-Szollosi, T. Kremmer, A. Malorni, and K. Vekey, J. Mass Spectrom., 40, 1472 (2005).

10) Y. Mechref and M. V. Novotny, Anal. Chem., 70, 455 (1998).

11) E. D. Green, G. Adelt, J. U. Baenziger, S. Wilson, and H. V. Halbeek, Biol. Chem., 264, 18253 (1988).

12) R. R. Townsend, M. R. Hardy, D. A. Cumming, J. P. Carver, and B. Bendiak, Anal. Biochem., 182, 1 (1989).

13) Y. O. Tsybin, P. Håkansson, B. A. Budnic, K. F. Haselmann, F. Kjeldsen, M. Gorshlov, and R. A. Zubarev,
Rapid Commun. Mass Spectrom., 15, 1849 (2001).

14) K. F. Haselmann, B. A. Budnic, J. V. Olsen, M. L. Nielsen, C. A. Reis, H. Clausen, A. H. Johnsen, and R. A. Zubarev, Anal. Chem., 73, 2998 (2001).

15) Y. O. Tsybin, O. Y. Tsybin, P. D. Grigoriev, M. R. Emmett, C. L. Hendrickson, and A. G. Marshall, Abstract Book of $17^{\text {th }}$ IMSC in Prague, ThP-007, 195 (2006).

16) N. Leymarie, C. E. Costello, and P. B. O'Connor, J. Am. Chem. Soc., 125, 8949 (2003).

17) M. A. Belyayev, J. J. Cournoyer, C. Lin, and P. B. O'connor, Am. Soc. Mass Spectrom., 10, 1428 (2006).

18) R. A. Zubarev, N. A. Kruger, E. K. Fridriksson, M. A. Lewis, D. M. Horn, B. K. Carpenter, and F. W. McLafferty, J. Am. Chem. Soc., 121, 2875 (1999).

19) E. Uggerud, Int. J. Mass Spectrom., 234, 45 (2004).

20) A. Sawicka, P. Skurski, R. R. Hudgins, and J. Simons, J. Phys. Chem. B, 107, 13505 (2003).

21) A. Al-Khalili, R. Thomas, A. Ehlerding, F. Hellberg, W. D. Geppert, V. Zhaunerchyk, M. af Ugglas, and M. Larsson, J. Chem. Phys., 121, 5700 (2004).

Keywords: $\quad$ ECD, $N$-Linked glycopeptide 